CHUNBO ZHANG, Ph.D. Candidate ${ }^{1}$

(Corresponding author)

E-mail: zhangchunbochn@yeah.net

XIUCHENG GUO, Ph.D. ${ }^{1}$

(Corresponding author)

E-mail: seuguo@163.com

ZHENPING XI, Master ${ }^{2}$

E-mail: 823844975@qq.com

${ }^{1}$ School of Transportation, Southeast University

No. 2 Sipailou, Nanjing 210096, China

2 Suzhou Planning \& Design Research Institute

No.747 Shiquan Street, Suzhou 215006, China
Traffic Engineering

Preliminary Communication

Submitted: 14 Mar. 2016

Accepted: 16 Nov. 2016

\title{
DETERMINATION OF OBSERVATION WEIGHT TO CALIBRATE FREEWAY TRAFFIC FUNDAMENTAL DIAGRAM USING WEIGHTED LEAST SQUARE METHOD (WLSM)
}

\begin{abstract}
Due to unbalanced speed-density observations, the one-regime traffic fundamental diagram and speed-density relationship models using least square method (LSM) cannot reflect actual conditions under congested/jam traffic. In that case, it is inevitable to adopt the weighted least square method (WLSM). This paper used freeway Georgia State Route 400 observation data and proposed 5 weight determination methods except the LSM to analyse 5 wellknown one-regime speed-density models to determine the best calibrating models. The results indicated that different one-regime speed-density models have different best calibrating models, for Greenberg, it was possible to find a specific weight using LSM, which is similar for Underwood and Northwestern Models, but different for that one known as 3PL model. An interesting case is the Newell's Model which fits well with two distinct calibration weights. This paper can make contribution to calibrating a more precise traffic fundamental diagram.
\end{abstract}

\section{KEY WORDS}

fundamental diagram; Weighted Least Square Method; observation weight; speed-density relationship;

\section{INTRODUCTION}

The speed and density relationship determines the traffic fundamental diagram [1-3], so it is extremely important to analyse the speed and density relationship and this has received much attention within the past few decades. Several models including single-regime and multiple-regime ones were proposed by researchers. Greenshields et al. [4] according to limited observations established the over-simplified linear model; Greenberg [5] developed a model by treating the traffic stream as a continuous fluid and the model turned out to be suitable for the traffic flow theory;
Underwood thought that the exponential relationship between the negative density and speed could be used in free-flow conditions and the Northwestern Model is similar but a little more complicated than the Underwood model [6]; Newell [7] considered the non-linear effects in the dynamics of car following and obtained a special model; Wang et al. [8] developed the logistic model of speed-density relationship motivated by the success of the generalized logistic curves in modelling the growth pattern phenomenon such as population dynamics, plant growth in agriculture, epidemic growth in biology, and market growth in economics. Besides, some multiple-regime models were also developed by Edie [9], Sun and Zhou [10], etc. Though multiple-regime models are more accurate than single-regime ones, they are not suggested to be used because of lack of mathematical elegance. Table 1 lists five wellknown single-regime models, each with equal to or less than three parameters [11].

Almost all the researchers calibrated the parameters of the single-regime models using the least square method (LSM) and Qu et al. [11] verified that there is deficiency of the least square method because of the unbalanced distribution of the observations in the light-traffic/free-flow conditions and congested/jam conditions. Then, the weighted least square method (WLSM) calibrating the single-regime models and one weight determination method were proposed.

However, is the only weight determination method proposed by Qu et al. [11] the best one? Are there any other better weight determination methods? Then, the main objective of this paper is to determine the suitable weight of WLSM to calibrate well the single-regime models.

The paper is organized as follows. The WLSM and methods of weight determination are provided in Section 2. The observation data information is 
Table 1 - Five well-known single-regime speed-density models

\begin{tabular}{||l|c|c||}
\hline \multicolumn{1}{|c|}{ Models } & Function & Parameters \\
\hline \hline Greenberg [5] & $v=v_{0} \ln \left(k_{j} / k\right)$ & $v_{0}, k_{j}$ \\
\hline Underwood [6] & $v=v_{f} \exp \left(-k / k_{0}\right)$ & $v_{f}, k_{0}$ \\
\hline Northwestern [6] & $v=v_{f} \exp \left\lfloor-0.5\left(k / k_{0}\right)^{2}\right\rfloor$ & $v_{f}, k_{0}$ \\
\hline Newell [7] & $v=v_{f}\left\{1-\exp \left\lfloor-\left(\eta / v_{f}\right)\left(1 / k-1 / k_{j}\right)\right]\right\}$ & $v_{f}, k_{j,} \eta$ \\
\hline Three-parameter logistic (3PL)model[8] & $v=v_{f} /\left\{1+\exp \left[\left(k-k_{0}\right) / \xi\right]\right\}$ & $v_{f}, k_{0}, \xi$ \\
\hline \hline
\end{tabular}

Note: $v$ - speed (the dependent variable); $v_{f}$-free-flow speed; $v_{0}$ - at-capacity speed; $k$ - density (the independent variable); $k_{j}$ - jam density; $k_{0}$ - at-capacity density; $\eta, \xi$ - coefficients

provided in Section 3. Section 4 presents the calibrating results with five weight determination methods and discusses the applicability of these methods. Finally, the conclusion is presented in Section 5.

\section{METHODOLOGY}

\subsection{Weighted least square method}

Since there is unbalanced distribution of the observations in the light-traffic/free-flow conditions and congested/jam conditions, the weighted least square method (WLSM) was proposed to calibrate the speed-density model parameters [11]. WLSM has been widely used in many fields by researchers: e.g. Veraart et al. used WLSM to estimate the diffusion MRI parameters [12]; Zhuang et al. proposed an improved meshless Shephard and WLSM possessing the delta property [13]; Fang did a complete analysis of the WLSM problem considering fixed and random parameters [14]; Mahboub and Sharifi developed a WLSM with linear and quadratic constraints [15]; Ciucci adopted WLSM to revisit parameter identification in electrochemical impedance spectroscopy [16]; Wang et al. used WLSM to make Multi-Gaussian fitting for pulse waveform [17]; Khatibinia et al. assessed seismic reliability of RC structures including soil-structure interaction using WLSM [18]; Parrish et al. used WLSM to analyse the acceleration of coupled cluster singles and doubles [19]; Stanley and Doucouliagos did WLSM meta-analysis for neither fixed nor random conditions [20]; and Einemo and So used WLSM for target localization in distributed MIMO radar [21].

Considering the $n$ speed-density data points $\left(k_{1}, v_{1}\right),\left(k_{2}, v_{2}\right), \ldots,\left(k_{n}, v_{n}\right)$ and one single-regime model function $v=f(k, b)$, where $b$ is an m-dimensional vector of parameters to be calibrated. To obtain the values of $\mathrm{b}$ based on WLSM, the sum of weighted squared errors are minimized mathematically as shown in Equation 1.

$\min S=\sum_{i=1}^{n} w_{i}\left[v_{i}-f\left(v_{i}, b\right)\right]^{2}$

where, $w_{i}$ is the weight of speed-density observation $i$. Since the single-regime model $v=f(k, b)$ is continuously differentiable, the first order partial derivative of $S$ to $b$ is zero when $S$ is minimum, that is

$$
\begin{aligned}
& \frac{\partial s}{\partial b_{j}}=2 \sum_{i=1}^{n} w_{i}\left[v_{i}-f\left(v_{i}, b\right)\right] \frac{\partial f\left(v_{i}, b\right)}{\partial b_{j}}=0, \\
& j=1,2, \ldots, m
\end{aligned}
$$

Then, the values of $b$ can be computed if each $w_{i}$ is known.

\subsection{Weight determination methods}

One weight determination method proposed by Qu et al. [11] is as follows.

Step 1: Rank the speed-density observations considering their densities. Data points become

$\left(v_{1}, k_{1}\right),\left(v_{2}, k_{2}\right), \ldots,\left(v_{i}, k_{i}\right), \ldots,\left(v_{n}, k_{n}\right)$

where, $k_{1} \leq k_{2} \leq \ldots \leq k_{i} \leq \ldots \leq k_{n}$ and $v_{i}$ is the corresponding speed.

Step 2: Let $\eta$ denote the maximum index $i$ that equals the same density as $k_{1}$, that is,

$\eta=\max \left\{i=1,2, \ldots, n \mid k_{i}=k_{1}\right\}$

Then,

$w_{i}=\frac{k_{\eta+1}-k_{1}}{\eta}, \quad i=1,2, \ldots, \eta$

Step 3: Let $\xi=\eta+1$. Let $\eta$ denote the maximum index $i$ that equals the same density as $k_{\xi}$, that is,

$\eta=\max \left\{i=\xi, \xi+1, \xi+2, \ldots, n \mid k_{i}=k \xi\right\}$

If $\xi<n$, then,

$w_{i}=\frac{k_{\eta+1}-k \xi-1}{2(\eta-\xi+1)}, \quad i=\xi, \xi+1, \xi+2, \ldots, \eta$

and repeat Step 3. Else,

$w_{i}=\frac{k_{n}-k_{\xi}-1}{n-\xi+1}, \quad i=\xi, \xi+1, \xi+2, \ldots, n$

and stop. 
However, the optimum weight might not be as shown above. Other four methods are proposed by this paper; the steps are the same as the above except Equations 5a, $7 a$ and 8 a. Those are:

$w_{i}=\left(\frac{k_{\eta+1}-k_{1}}{\eta}\right)^{\frac{1}{3}}, \quad i=1,2, \ldots, \eta$

$w_{i}=\left[\frac{k_{\eta+1}-k_{\xi-1}}{2(\eta-\xi+1)}\right]^{\frac{1}{3}}, \quad i=\xi, \xi+1, \xi+2 \ldots, \eta$

$w_{i}=\left(\frac{k_{n}-k_{\xi-1}}{\eta-\xi+1}\right)^{\frac{1}{3}}, \quad i=\xi, \xi+1, \xi+2 \ldots, n$

$w_{i}=\left(\frac{k_{\eta}+1-k_{1}}{\eta}\right)^{\frac{1}{2}}, \quad i=1,2, \ldots, \eta$

$W_{i}=\left[\frac{k_{\eta+1}-k \xi-1}{2(\eta-\xi+1)}\right]^{\frac{1}{2}}, \quad i=\xi, \xi+1, \xi+2 \ldots, \eta$

$w_{i}=\left(\frac{k_{n}-k_{\xi-1}}{\eta-\xi+1}\right)^{\frac{1}{2}}, \quad i=\xi, \xi+1, \xi+2 \ldots, n$

$w_{i}=\left(\frac{k_{\eta+1}-k_{1}}{\eta}\right)^{2}, \quad i=1,2, \ldots, \eta$

$w_{i}=\left[\frac{k_{\eta+1}-k_{\xi-1}}{2(\eta-\xi+1)}\right]^{2}, \quad i=\xi, \xi+1, \xi+2 \ldots, \eta$

$w_{i}=\left(\frac{k_{n}-k_{\xi-1}}{\eta-\xi+1}\right)^{2}, \quad i=\xi, \xi+1, \xi+2 \ldots, n$

$w_{i}=\left(\frac{k_{\eta+1}-k_{1}}{\eta}\right)^{3}, \quad i=1,2, \ldots, \eta$

$w_{i}=\left[\frac{k_{\eta+1}-k \xi-1}{2(\eta-\xi+1)}\right]^{3}, \quad i=\xi, \xi+1, \xi+2 \ldots, \eta$

$w_{i}=\left(\frac{k_{n}-k \xi-1}{\eta-\xi+1}\right)^{3}, \quad i=\xi, \xi+1, \xi+2 \ldots, n$

The reason why the above weight determination methods are selected is that they reflect different relationship between the weight and density interval and they can simply represent much complicated conditions. The specific shape of these five weight determination methods is intuitively shown in Figure 1, which reflects the different characteristics of these five weight determination methods. Then, these five weight determination methods and LSM are used to calibrate the speed-density model parameters.

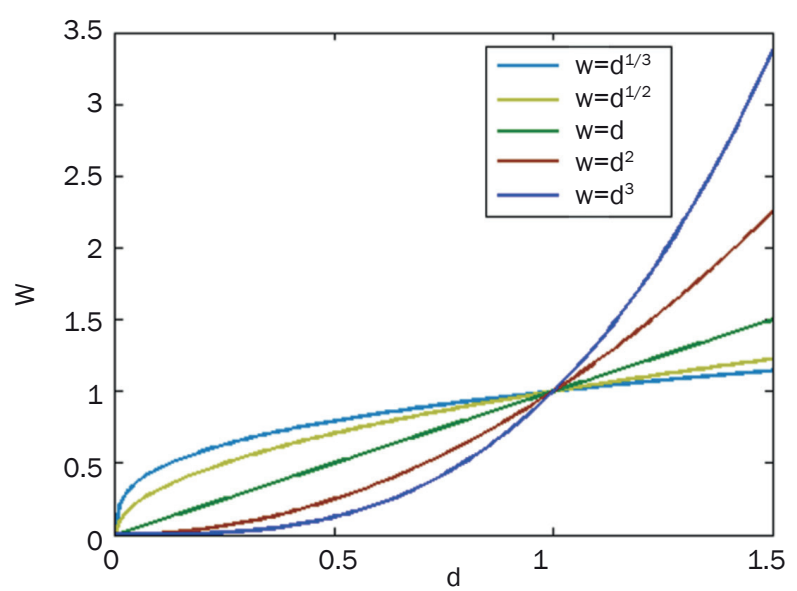

Figure 1 - Weight and density interval relationship of five weight determination methods

\subsection{Relative error (RE) and root mean square error (RMSE)}

To validate which method is suitable to calibrate the speed-density model accurately, relative error (RE) [11] and root mean square error (RMSE) [22] are used, and those are:

$R E=\frac{1}{n} \sum_{i=1}^{n} \frac{\left|v_{i}-\widehat{v}_{i}\right|}{v_{i}}$

RMSE $=\sqrt{\frac{1}{n}\left(v_{i}-\widehat{v}_{i}\right)^{2}}$

where $v_{i}$ is the actual speed of observation $i$ and $\widehat{v}_{i}$ is the predicted speed of the calibrated model for observation $i$.

\section{OBSERVATION DATA INFORMATION}

The original Georgia State Route 400 (GA400) ITS data were aggregated every 5 minutes, which were often used to generate the fundamental diagrams [8]. GA400 is a controlled-access state highway in the northern part of the US state of Georgia and the data were collected from a section with four lanes for one direction. One-year 44,787 continuous observations were obtained in 2003 , out of which the time interval is long enough to calibrate the speed-density models. The specific distribution of these data is shown in Figure 2 and in Table 2 and the unbalanced distribution of observation distribution can be easily seen.

Table 2 - Frequencies of GA400 speed-density data

\begin{tabular}{|l|c|c|c|c|c|c|c||}
\hline Density [veh/km] & $0-10$ & $10-20$ & $20-30$ & $30-40$ & $40-50$ & $50-60$ & $60-70$ \\
\hline \hline Frequencies & 9,333 & 29,329 & 2,665 & 1,105 & 827 & 529 & 346 \\
\hline \hline Density [veh/km] & $70-80$ & $80-90$ & $90-100$ & $100-110$ & $110-120$ & $120-130$ & $130-140$ \\
\hline \hline Frequencies & 268 & 173 & 136 & 48 & 21 & 6 & 1 \\
\hline
\end{tabular}




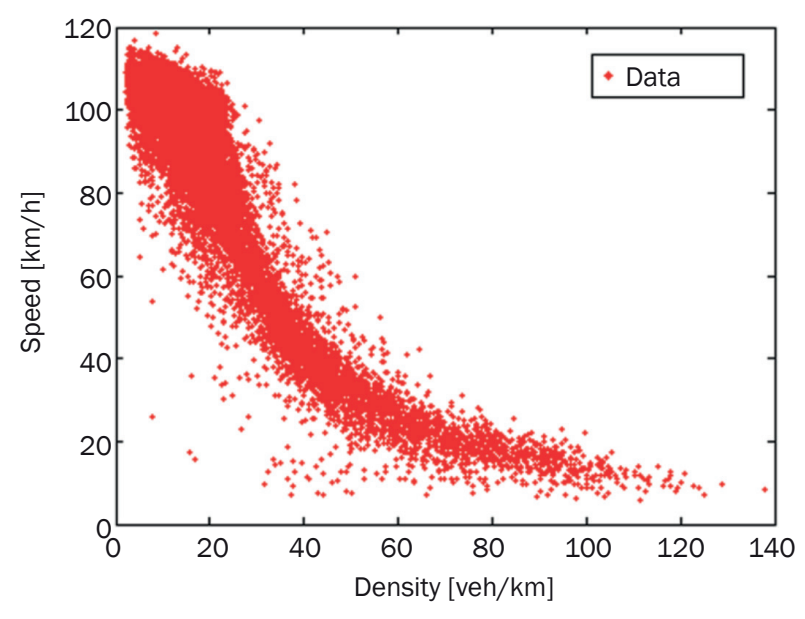

Figure 2 - Data distribution of GA400

\section{RESULTS AND DISCUSSION}

The calibrating results of five well-known single-regime models with five weight determination methods and LSM are shown in Figure 3 and Table 3.

From Figure 3, it is obvious that five calibrating models using LSM can reflect the speed-density relationship under the light-traffic/free-flow traffic (when the density is less than $30 \mathrm{veh} / \mathrm{km}$ ). However, all models with LSM cannot reflect the speed-density relationship under congested/jam conditions (when the density is more than $60 \mathrm{veh} / \mathrm{km}$ ), except the Underwood Model. Then, the five calibrating models using WLSM with five different weight determination methods can reflect the speed-density relationship better, especially under congested/jam conditions. In order to make sure which weight determination method is the best, the RE and RMSE of five models under five weight determination methods and LSM are obtained in Figure 4 and Figure 5, respectively.

From Figure 4 and Figure 5, for Greenberg [5] Model, the calibrating model using LSM is not suitable because of the large values of RE and RMSE under congested/jam conditions, and the other five calibrating models are better under congested/jam conditions, especially the two calibrating models with weights $w_{2}$ and $w_{3}$. However, the two models cannot indicate the precise relationship between the speed and density under light-traffic/free-flow traffic; REs and RMSEs are over 0.3 and 30, respectively, when the density is less than $30 \mathrm{veh} / \mathrm{km}$. The other three calibrating models are similar and they can all reflect the speed-density relationship; the REs and RMSEs of the three calibrating models are all below 0.2 and 20 , respectively, when the density is less than $30 \mathrm{veh} / \mathrm{km}$, and under congested/jam conditions they are all prior to the model using LSM. By comparison, the calibrating model with weight $w_{1}$ is better than the other two models overall and it is the best model to determinate the speed-density relationship.

For Underwood Model [6], the calibrating model using LSM cannot also reflect the precise speed-density relationship under congested conditions, and the other five calibrating models are better when the density is more than $40 \mathrm{veh} / \mathrm{km}$, except when the density is more than 100 veh $/ \mathrm{km}$, which might be due to the fewer points with only 76 observations. Though the two calibrating models with weights $w_{2}$ and $w_{3}$ can reflect the speed-density relationship precisely under congested/jam conditions, they cannot reflect the relationship under light-traffic/free-flow traffic, and REs and RMSEs of the two models are more than 0.3 and 25, respectively, when the density is less than $30 \mathrm{veh} / \mathrm{km}$. The other three calibrating models with weights $w_{1}, w_{1 / 3}$ and $w_{1 / 2}$ are similar and all can reflect the

Table 3 - Calibrating values of parameters of five well-known single-regime models with different weights

\begin{tabular}{|c|c|c|c|c|c|c|}
\hline & $w_{0}$ & $w_{1}$ & $w_{1 / 3}$ & $W_{1 / 2}$ & $w_{2}$ & $w_{3}$ \\
\hline \multirow{2}{*}{$v=v_{0} \ln \left(k_{j} / k\right)$} & $v_{0}=30.88$ & $v_{0}=35.50$ & $v_{0}=36.01$ & $v_{0}=37.17$ & $v_{0}=22.34$ & $v_{0}=14.95$ \\
\hline & $k_{j}=291.0$ & $k_{j}=148.8$ & $k_{j}=173.5$ & $k_{j}=154.2$ & $k_{j}=197.9$ & $k_{j}=242.7$ \\
\hline \multirow{2}{*}{$v=v_{f} \exp \left(-k / k_{0}\right)$} & $v_{f}=129.3$ & $v_{f}=129.6$ & $v_{f}=132.1$ & $v_{f}=132.7$ & $v_{f}=80.25$ & $v_{f}=47.15$ \\
\hline & $k_{0}=47.60$ & $k_{0}=40.24$ & $k_{0}=42.40$ & $k_{0}=40.88$ & $k_{0}=60.03$ & $k_{0}=80.22$ \\
\hline \multirow{2}{*}{$v=v_{f} \exp \left\lfloor-0.5\left(k / k_{0}\right)^{2}\right\rfloor$} & $v_{f}=109.5$ & $v_{f}=100.5$ & $v_{f}=108.7$ & $v_{f}=107.9$ & $v_{f}=36.15$ & $v_{f}=20.97$ \\
\hline & $k_{0}=31.06$ & $k_{0}=35.44$ & $k_{0}=31.43$ & $k_{0}=31.88$ & $k_{0}=79.01$ & $k_{0}=102.3$ \\
\hline \multirow{3}{*}{$v=v_{f}\left\{1-\exp \left\lfloor-\left(\eta / v_{f}\right)\left(1 / k-1 / k_{j}\right)\right]\right\}$} & $v_{f}=106.8$ & $v_{f}=112.1$ & $v_{f}=108.2$ & $v_{f}=109.0$ & $v_{f}=118.3$ & $v_{f}=124.2$ \\
\hline & $\eta=4,573$ & $\eta=3,131$ & $\eta=4,110$ & $\eta=3,863$ & $\eta=2,289$ & $\eta=2,076$ \\
\hline & $k_{j}=98.36$ & $k_{j}=174.5$ & $k_{j}=113.3$ & $k_{j}=123.7$ & $k_{j}=287.0$ & $k_{j}=329.9$ \\
\hline \multirow{3}{*}{$v=v_{f} /\left\{1+\exp \left[\left(k-k_{0}\right) / \xi\right]\right\}$} & $v_{f}=124.8$ & \multirow{3}{*}{-} & $v_{f}=142.3$ & $v_{f}=161.8$ & \multirow{3}{*}{-} & \multirow{3}{*}{-} \\
\hline & $k_{0}=33.10$ & & $k_{0}=28.28$ & $k_{0}=22.39$ & & \\
\hline & $\xi=14.40$ & & $\xi=18.48$ & $\xi=21.59$ & & \\
\hline
\end{tabular}

For Wang et al. [8] 3PL Model, the calibrating values of parameters with significance 0.05 under weights $w_{1}, w_{2}$ and $w_{3}$ cannot be obtained through calculation, so only $w_{0}, w_{1 / 3}$ and $w_{1 / 2}$ are indicated in Table 3. 


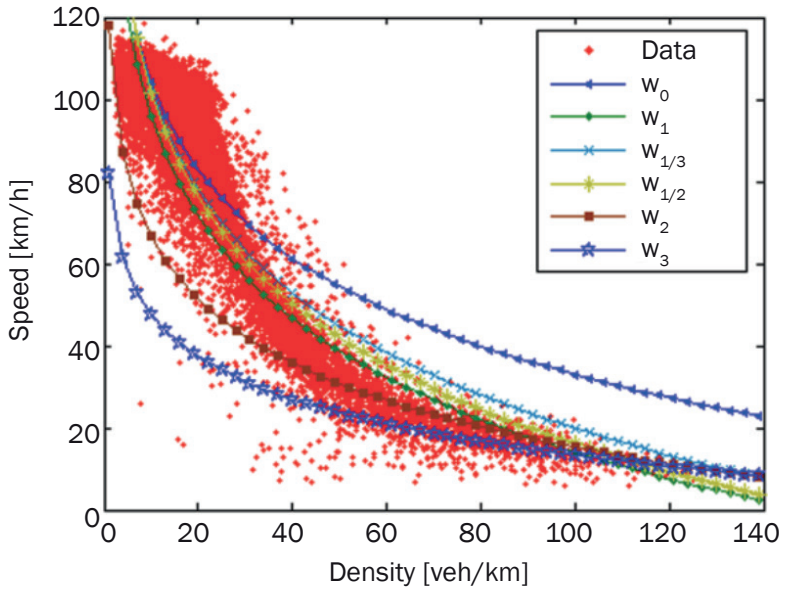

a) Greenberg Model

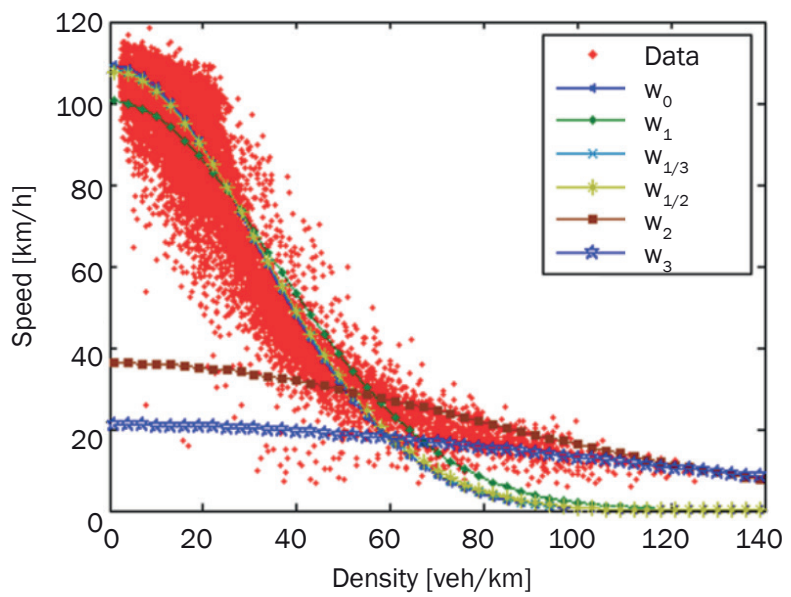

c) Northwestern Model

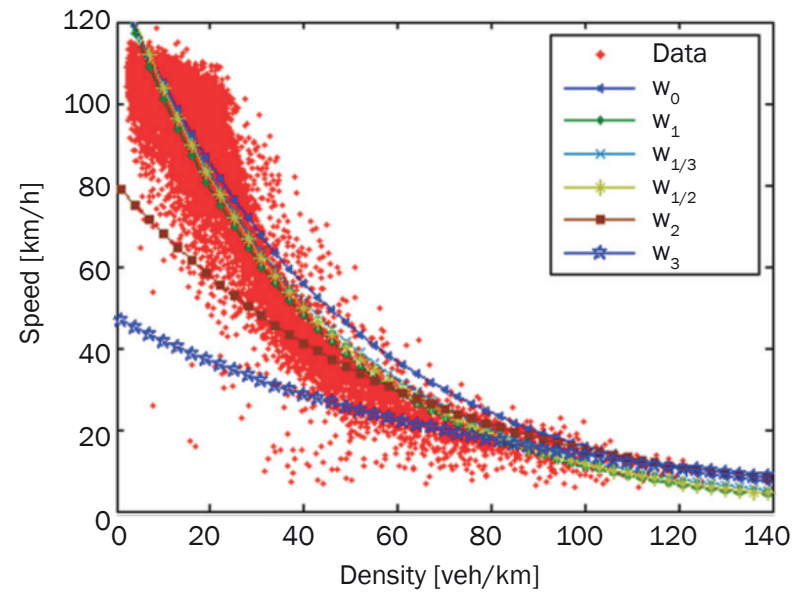

b) Underwood Model

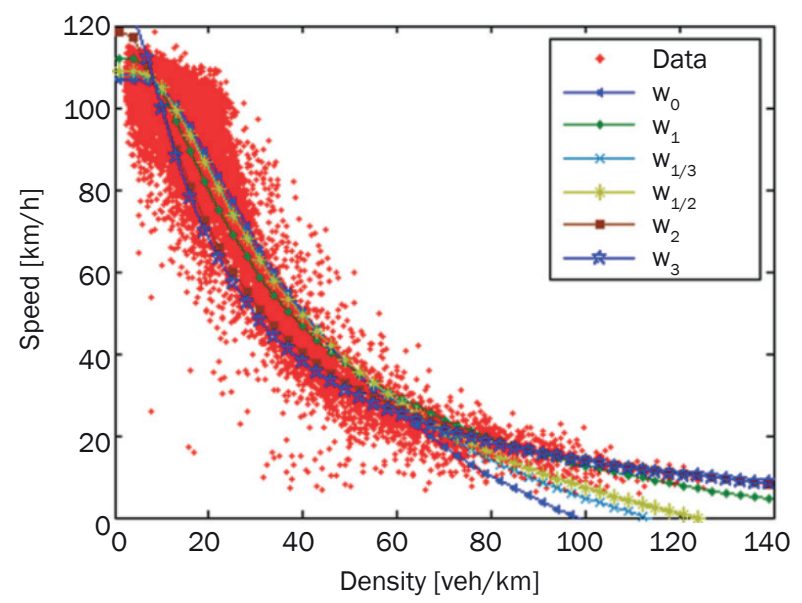

d) Newell Model

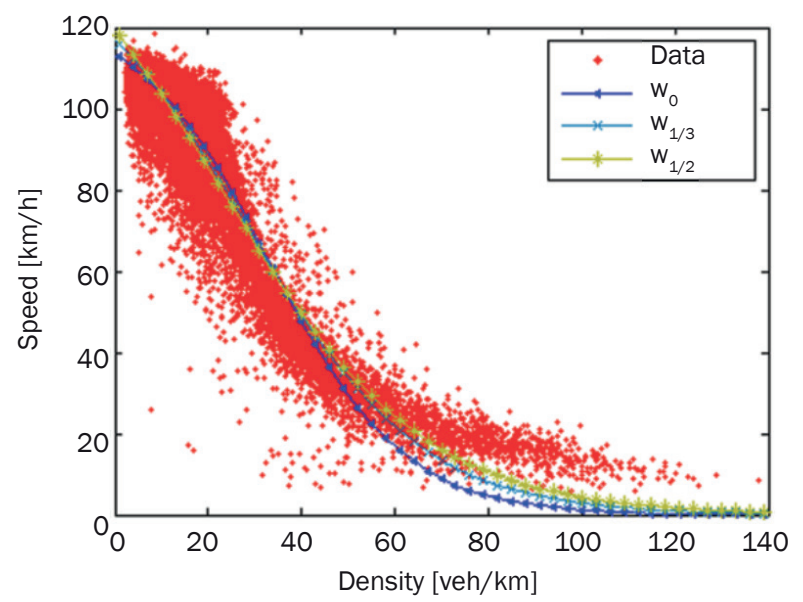

e) Wang et al. [8] 3PL Model

Figure 3 - Calibrating results of different models with different weights

Notes: $w_{0}$ means LSM, $w_{1}$ means the weight determination method of Equations $5 a$, $7 a$ and $8 a, w_{1 / 3}$ means that of Equations $5 b, 7 b$ and $8 b$, etc. For Wang et al. [8] 3PL Model, the calibrating values of parameters with significance 0.05 under weights $w_{1}, w_{2}$ and $w_{3}$ cannot be obtained through calculation, so only $w_{0}, w_{1 / 3}$ and $w_{1 / 2}$ are indicated in Figure $3 e$.

speed-density relationship; REs and RMSEs of the three calibrating models are all below 0.35 and 13 , respectively, both under congested/jam conditions and light-traffic/free-flow traffic. By comparison, the calibrating model with weight $w_{1}$ is better than the other two models overall and it is the best model to determinate the speed-density relationship.

For Northwestern Model [6], the calibrating model using LSM is not suitable because of large values of $\mathrm{RE}$ and RMSE under congested/jam conditions, and 


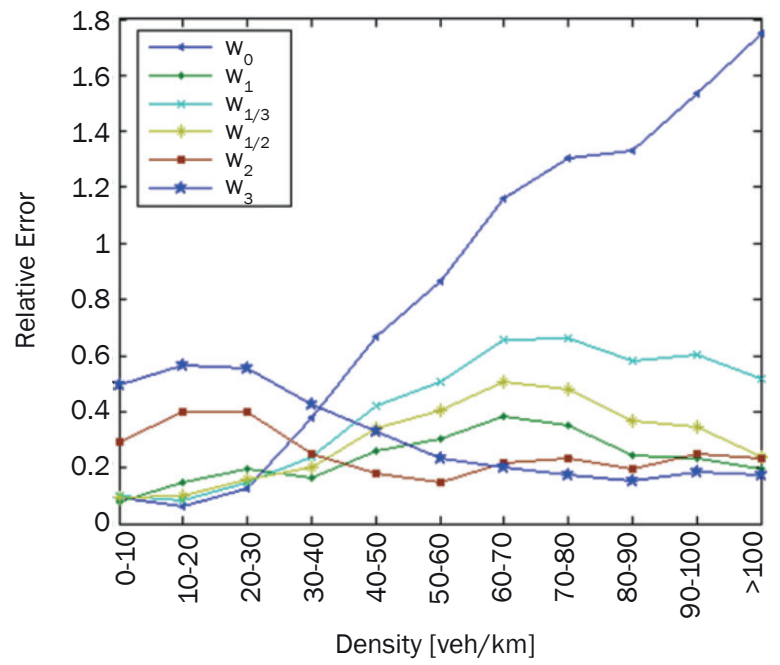

a) REs of Greenberg Model

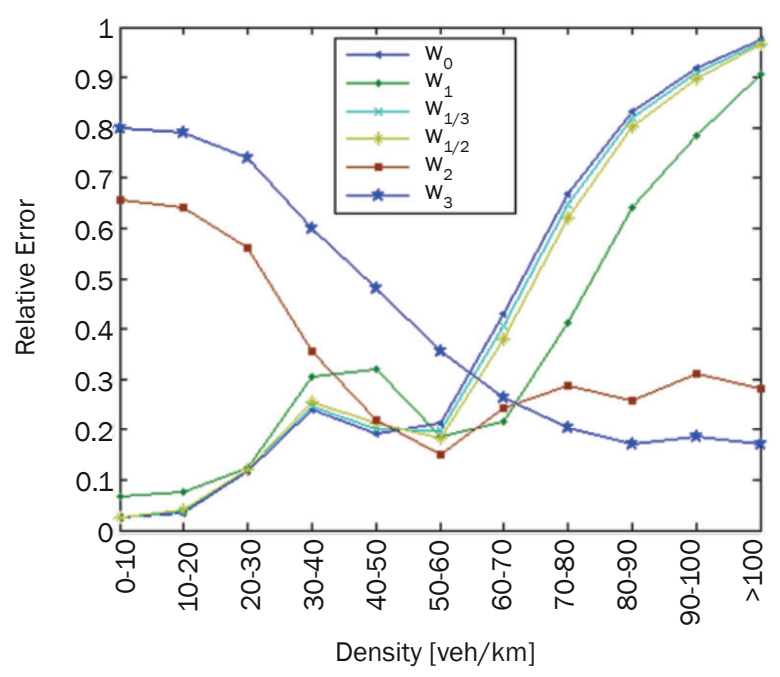

c) REs of Northwestern Model

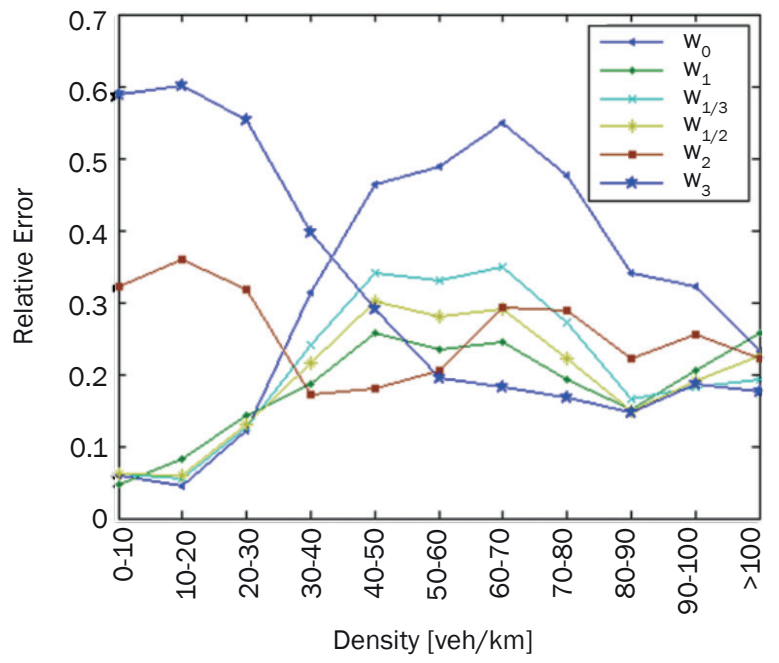

b) REs of Underwood Model

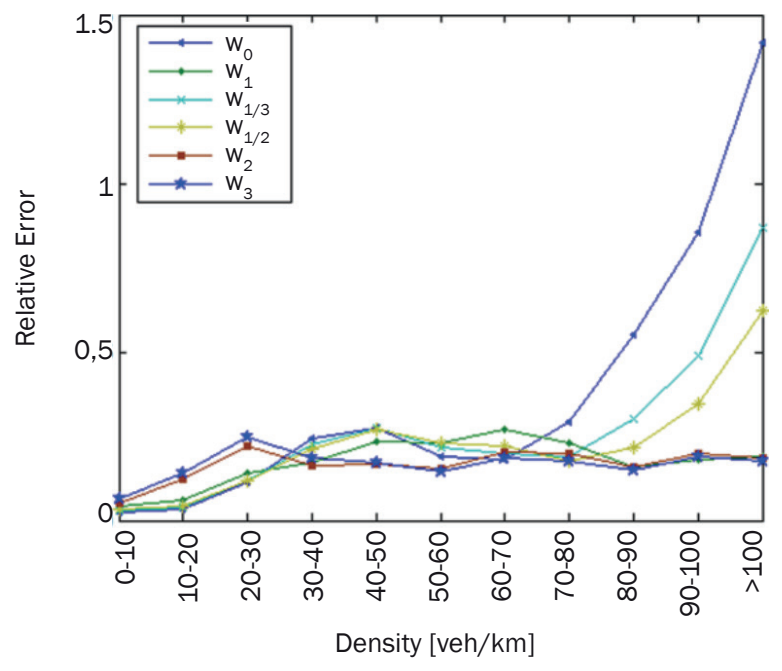

d) REs of Newell Model

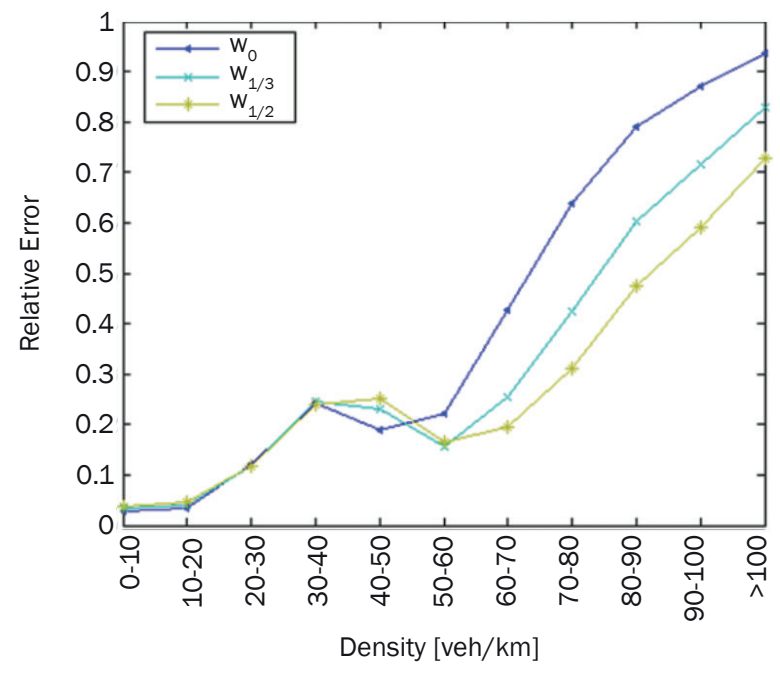

e) REs of Wang et al. [8] 3PL Model

Figure 4 - REs of five calibrating models under different conditions 


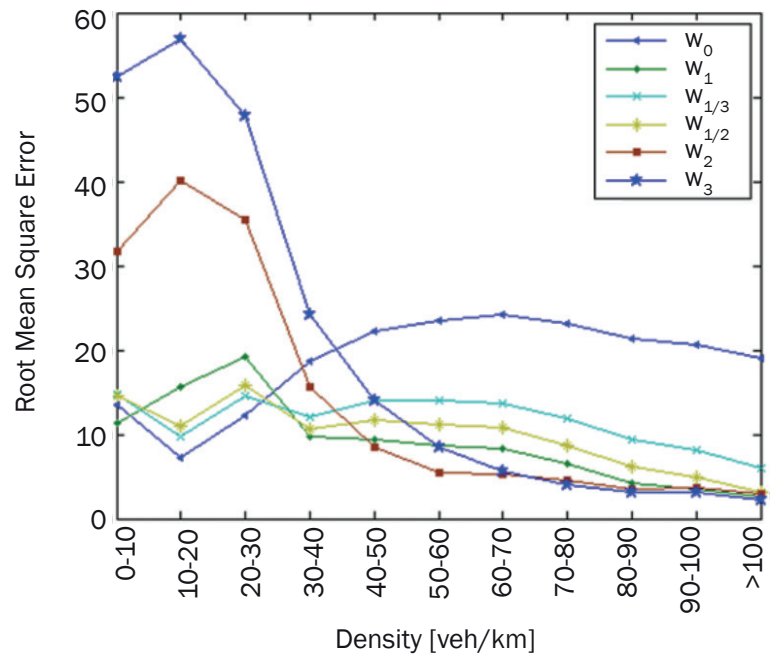

a) RMSEs of Greenberg Model

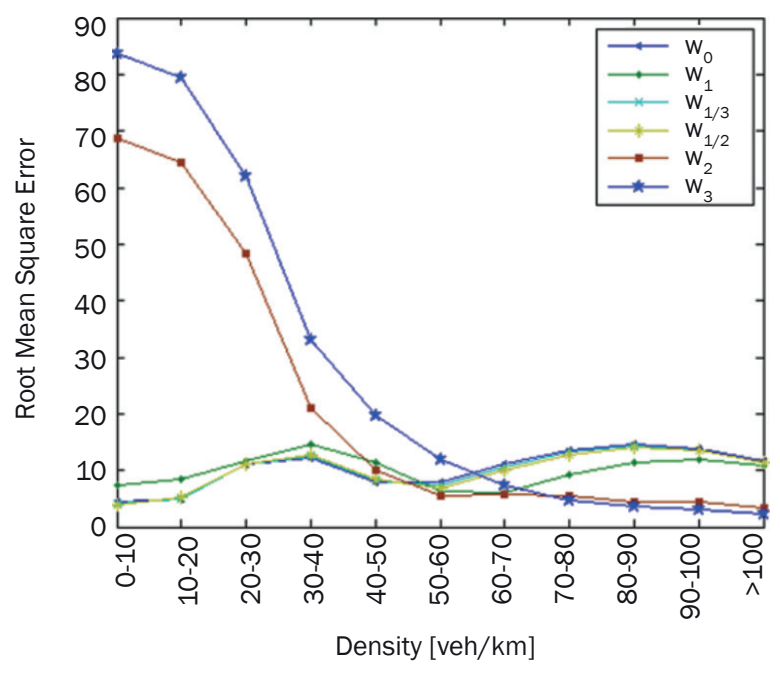

c) RMSEs of Northwestern Model

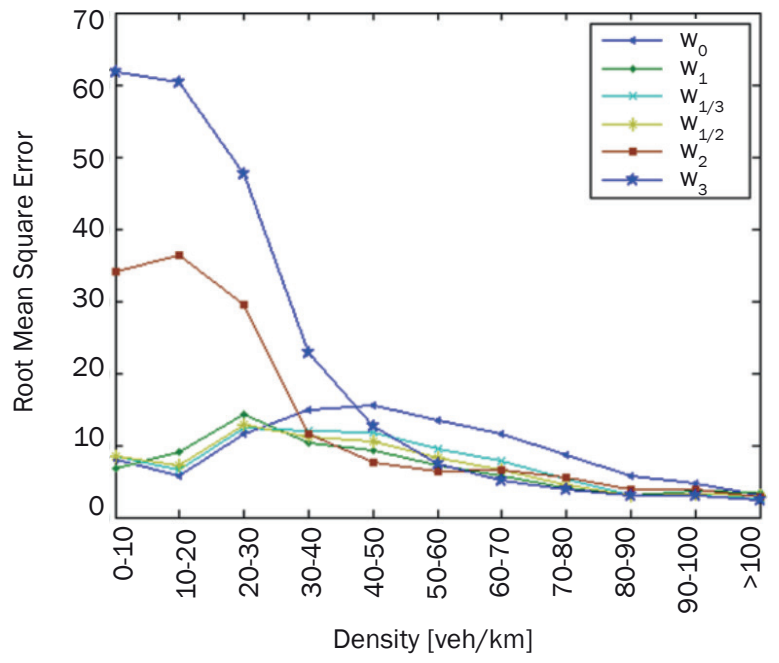

b) RMSEs of Underwood Model

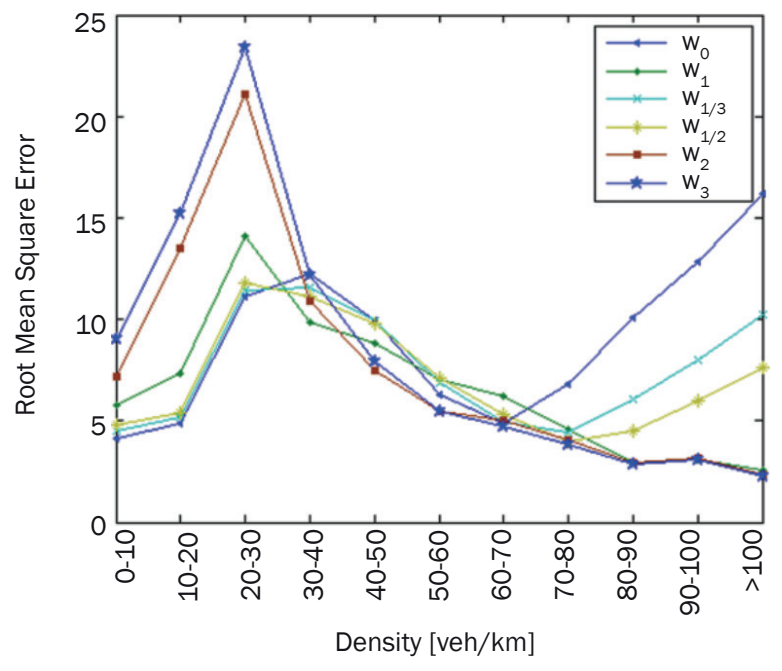

d) RMSES of Newell Model

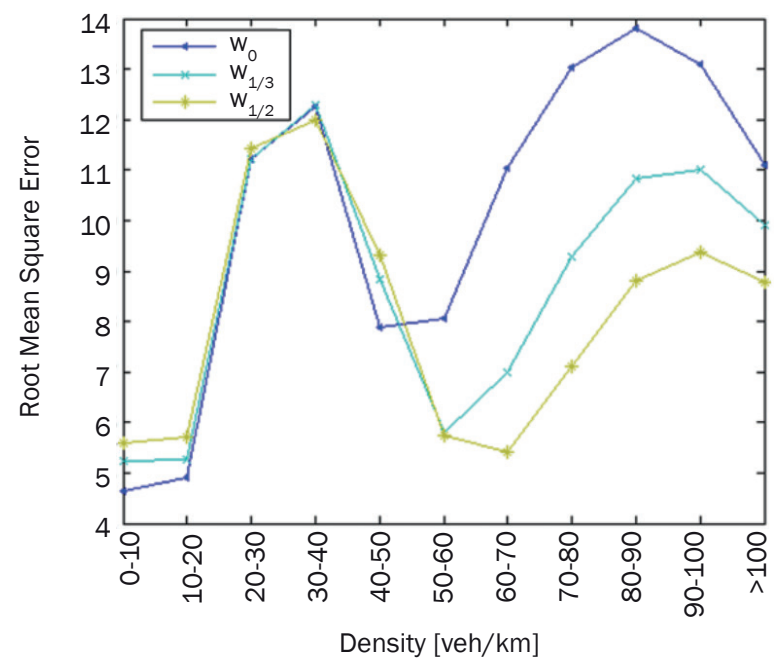

e) RMSEs of Wang et al. [8] 3PL Model

Figure 5 - RMSEs of five calibrating models under different conditions 
the other five calibrating models are better when the density is more than $60 \mathrm{veh} / \mathrm{km}$. The two calibrating models with weights $w_{2}$ and $w_{3}$ can reflect the speed-density relationship precisely when the density is more than $70 \mathrm{veh} / \mathrm{km}$ while the two models cannot reflect the relationship under light-traffic/free-flow traffic and REs and RMSEs of the two models are more than 0.5 and 45 , respectively, when the density is less than $30 \mathrm{veh} / \mathrm{km}$. The two models with weights $w_{1 / 3}$ and $w_{1 / 2}$ are very similar with the model using LSM, which cannot all reflect the speed-density precisely. As for the calibrating model with weight $w_{1}$, it can reflect the speed-density relationship precisely overall and it is the best model to determinate the speed-density relationship.

For Newell [7] Model, the calibrating model using LSM cannot also reflect the precise speed-density relationship under congested/jam conditions, and the other five calibrating models are better when the density is more than 70 veh $/ \mathrm{km}$. The two calibrating models with weights $w_{1 / 3}$ and $w_{1 / 2}$ cannot reflect the speed-density relationship more precisely than the calibrating model with weight $w_{1}$ as a whole, especially when the density is more than 80 veh $/ \mathrm{km}$. Besides, the calibrating model with weight $w_{3}$ is not as precise as the calibrating model with weight $w_{2}$, especially when the density is less than $50 \mathrm{veh} / \mathrm{km}$. As for the two calibrating models with weighs $w_{2}$ and $w_{1}$, they can both reflect the speed-density relationship precisely overall and they both are the best calibrating models. If the speed-density relationship under congested/jam conditions is stressed, the calibrating model with weight $w_{2}$ is the best one; on the contrary, if the relationship is under light-traffic/free-flow traffic, the calibrating model with weight $w_{1}$ is the best one.

For Wang et al. [8] 3PL Model, though the above calibrating models with weight $w_{1}$ are almost the best calibrating models, the calibrating Wang et al. [8] 3PL Model with weight $w_{1}$ with significance 0.05 cannot be obtained according to calculation, nor are the two calibrating models with weights $w_{2}$ and $w_{3}$. For these calibrating models with LSM, weights $w_{1 / 3}$ and $w_{1 / 2}$, are all similar, especially when the density is less than $50 \mathrm{veh} / \mathrm{km}$. However, the two calibrating models with weighs $w_{1 / 3}$ and $w_{1 / 2}$ are significantly better than the one with LSM when the density is more than 50 veh/ $\mathrm{km}$. Finally, by comparison, the calibrating model with weight $w_{1 / 2}$ is the best one.

The reason why different speed-density models have different best weight determination methods is that different speed-density models can reflect the speed-density relationship precisely under different conditions. All these models can indicate the specific speed-density relationship using LSM under light-traffic/free-flow traffic, but under congested/jam conditions, the precision is low. However, the lower precision of these models is not all when the density values are very large. For the Underwood Model, the density of the lowest precision of calibrating model using LSM in terms of RE is between 60 veh/ km and 70 veh/km; for the Newell [7] Model, the density of lower precision of calibrating models using LSM in terms of RMSEs is between $30 \mathrm{veh} / \mathrm{km}$ and $40 \mathrm{veh} / \mathrm{km}$, and more than $90 \mathrm{veh} / \mathrm{km}$; for Wang et al. [8] 3PL Model, the density of the lowest precision of calibrating models using LSM in terms of RMSE is between 80 veh/ $\mathrm{km}$ and 90 veh/km. Different weight determination methods can change these conditions, for Underwood Model, the density of the lowest precision of calibrating model with weight $w_{3}$ in terms of RE is between 10 veh $/ \mathrm{km}$ and $20 \mathrm{veh} / \mathrm{km}$; for the Newell [7] Model, the density of lower precision of calibrating models with weight $w_{3}$ in terms of RMSEs is between 20 veh $/ \mathrm{km}$ and 30 veh/km; for Wang et al. [8] 3PL Model, the density of the lowest precision of calibrating models with weight $w_{1 / 3}$ in terms of RMSE is between $30 \mathrm{veh} / \mathrm{km}$ and 40 veh/km. Then, according to both REs and RMSEs under different conditions, the different best observation weight method is determined.

\section{CONCLUSION}

This paper creatively analysed five well-known single-regime speed-density relationship models with five weight determination methods and LSM to use REs and RMSEs to find the best calibrating models. The results indicated that different models have different best calibrating models, for the Greenberg [5] Model, the calibrating model with weight $w_{1}$ is the best one, so are Underwood Model and Northwestern Model; for the Newell [7] Model, both calibrating models with weights $w_{1}$ and $w_{2}$ are the best ones; for Wang et al. [8] 3PL Model, the best one is the calibrating model with weight $w_{1 / 2}$, of which the calibrating models with weights $w_{2}$ and $w_{3}$ cannot be computed.

This paper has confirmed that the calibrating models using LSM cannot reflect the speed-density relationship under congested/jam conditions and the results indicated that different speed-density models need to use different weight determination methods and not the weight $w_{1}$ only. Then the more precise traffic fundamental diagram can be determined.

However, this paper only analysed the one-regime models, and the multi-regime models may not precisely reflect the speed-density relationship under certain conditions. In that case, the related studies should also be done on calibrating multi-regime models.

\section{ACKNOWLEDGEMENTS}

The authors are grateful to editors and reviewers for their suggestions and comments about this paper, and to the authors of cited papers for their contribution. 
We would also like to express our gratitude to Dr. Daiheng $\mathrm{Ni}$, Associate Professor, Department of Civil and Environmental Engineering, University of Massachusetts Amherst, for generously supplying the data needed in this paper.

张春波, 博士研究生 ${ }^{1}$

邮箱: zhangchunbochn@yeah. net

过秀成, 教授, 博士 1

邮箱: seuguo@163.com

奚振平, 硕士 ${ }^{2}$

邮箱: 823844975@qq. com

1 东南大学交通学院

中国南京市四牌楼 2 号, 邮编： 210096

2 苏州规划设计研究院

中国苏州市十全街747号，邮编：215006

\section{加权最小二乘法标定高速公路交通 基本图的权重确定方法研究}

\section{摘要}

由于实际速度一密度观测值的不均衡分布，采用 最小二乘法标定的单段式交通基本图和速度一密度 关系模型不能够准确反映拥堵条件下的实际交通状 况, 这种情况需要采用加权最小二乘法。本文采用 美国佐治亚州 400 号高速公路观测数据, 提出除了 最小二乘法外的 5 种权重确定方法，分析5个著名的 单段式速度一密度模型, 以达到准确标定结果的目 的。结果表明，不同的单段式速度一密度模型具有不 同的最佳标定权重，Greenberg模型采用加权最小 二乘法标定时具有特定的权重确定方法，Underwood 模型和Northwestern模型也具有相似的权重确定方 法但与三参数生长曲线模型有所不同。值得注意的 是, Newe 11 模型标定时具有两种不同的权重确定方 法。本文的研究对于更加准确标定交通基本图具有 一定贡献。

\section{关键词}

基本图；加权最小二乘法；观测权重 ; 速度一密度关系

\section{REFERENCES}

[1] Ambarwati L, Pel AJ, Verhaeghe R, van Arem B. Empirical analysis of heterogeneous traffic flow and calibration of porous flow model. Transp Res Part C:Emerg Technol. 2014;48:418-436. doi:10.1016/ j.trc.2014.09.017

[2] Antoniou C, Koutsopoulos HN, Yannis G. Dynamic data-driven local traffic state estimation and prediction. TranspRes Part C: Emerg Technol. 2013;34:89-107. doi:10.1016/j.trc.2013.05.012

[3] Ngoduy D, Maher MJ. Calibration of second order traffic models using continuous cross entropy method. Transp Res Part C: Emerg Technol. 2012;24:102-121. doi:10.1016/j.trc.2012.02.007

[4] Greenshields BD, Bibbins JR, Channing WS, Miller, HH.A study of traffic capacity. Highway Research Board. 1935;14:448-477.
[5] Greenberg H. An analysis of traffic flow. Oper Res. 1959;7(1):79-85. doi: 10.1287/opre.7.1.79

[6] Heydecker BG, Addison JD. Analysis and modelling of traffic flow under variable speed limits. Transp Res Part C: Emerg Technol. 2011;19(2):206-217. doi: 10.1016/j.trc.2010.05.008

[7] Newell GF. Nonlinear effects in the dynamics of car following. Oper Res. 1961;9(2):209-229.

[8] Wang $\mathrm{H}$, Li H, Chen QY, Ni D. Logistic modeling of the equilibrium speed-density relationship. Transp Res Part A: Policy Pract. 2011;45(6):554-566. doi:10.1016/j.tra.2011.03.010

[9] Edie LC. Car-following and steady-state theory for noncongested traffic. Oper Res. 1961;9:66-76.

[10] Sun L, Zhou J. Development of multiregime speeddensity relationship by cluster analysis. Transp Res Rec J Transp Res Board. 2005;193:64-71. doi: http:// dx.doi.org/10.3141/1934-07

[11] Qu X, Wang S, Zhang J. On the fundamental diagram for freeway traffic: A novel calibration approach for single-regime models. Transp Res Part B: Method. 2015;73:91-102. doi:10.1016/j.trb.2015.01.001

[12] Veraart J, Sijbers J, Sunaert S, Leemans A, Jeurissen B. Weighted linear least squares estimation of diffusion MRI parameters: strengths, limitations, and pitfalls. Neuroimage. 2013;81:335-346. doi:10.1016/ j.neuroimage.2013.05.028

[13] Zhuang X, Zhu H, Augarde C. An improved meshless Shepard and least squares method possessing the delta property and requiring no singular weight function. Comput Mech. 2014;53(2):343-357. doi: 10.1007/s00466-013-0912-1

[14] Fang X. Weighted total least squares: necessary and sufficient conditions, fixed and random parameters. J Geodesy. 2013;87(8):733-749. doi: 10.1007/s00190013-0643-2

[15] Mahboub V, Sharifi MA. On weighted total leastsquares with linear and quadratic constraints. J Geodesy. 2013;87(3):279-286. doi:10.1007/s00190-0120598-8

[16] Ciucci F. Revisiting parameter identification in electrochemical impedance spectroscopy: Weighted least squares and optimal experimental design. Electrochim Acta. 2013;87:532-545. doi:10.1016/j.electacta.2012.09.073

[17] Wang L, Xu L, Feng S, Meng MQH, Wang K. Multi-Gaussian fitting for pulse waveform using weighted least squares and multi-criteria decision making method. Comput Biol Med. 2013;43(11):1661-1672. doi:10.1016/j.compbiomed.2013.08.004

[18] Khatibinia M, Fadaee MJ, Salajegheh J, Salajegheh E. Seismic reliability assessment of RC structures including soil-structure interaction using wavelet weighted least squares support vector machine. Reliab Eng Syst Safe. 2013;110:22-33. doi:10.1016/ j.ress.2012.09.006

[19] Parrish RM, Sherrill CD, Hohenstein EG, Kokkila SI, Martínez TJ. Communication: Acceleration of coupled cluster singles and doubles via orbital-weighted least-squares tensor hypercontraction. J Chem Phys. 2014;140(18). doi:10.1063/1.4876016 
[20] Stanley TD, Doucouliagos H. Neither fixed nor random: weighted least squares meta-analysis. Stat Med. 2015;34(13):2116-2127. doi:10.1002/sim.6481

[21] Einemo M, So HC. Weighted least squares algorithm for target localization in distributed MIMO radar.
Signal Processing. 2015;115:144-150. doi: 10.1016/ j.sigpro.2015.04.004

[22] Washington SP, Karlaftis MG, Mannering FL. Statistical and econometric methods for transportation data analysis. London: Chapman and Hall; 2013. 